\title{
A Phase II Study of S-1 in Elderly Patients with Unresectable Pancreatic Cancer
}

\author{
Hitoshi Kuriyama ${ }^{1}$, Masahiko Inamori², Hiroshi lida², Koji Fujita², Akihiko Kusakabe², \\ Hidenori Ohkubo ${ }^{1}$, Eiji Sakai ${ }^{3}$, Yoriko Murata ${ }^{1}$, Shin Maeda ${ }^{4}$, Atsushi Nakajima ${ }^{3}$, Yasuo Hata ${ }^{1}$
}

'Department of Gastroenterology, Chigasaki Municipal Hospital, Kanagawa, Japan

2Department of Medical Education, Yokohama City University School of Medicine, Yokohama, Japan

${ }^{3}$ Department of Hepatology and Gastroenterology, Yokohama City University Hospital, Yokohama, Japan

${ }^{4}$ Department of Gastroenterology, Yokohama City University Hospital, Yokohama, Japan

\section{ABSTRACT}

Background/Aims: The aim of this study was to investigate the efficacy and safetyof the oral fluoropyrimidine derivativeS-1 in elderly patients with unresectable pancreatic cancer.

Methods: Patients were eligible if they were at least 70 years old and had unresectable pancreatic carcinoma. S-1 was administered orally twice daily after meals at doses of 80 , 100 , and $120 \mathrm{mg} /$ day forbody surface areasfor 28 consecutive days, followed by a 14-day rest period. Administration was repeated until the appearance of disease progression or unacceptable toxicity.

Results: Twenty-one patients were enrolledin this study. Five patients (23.8\%) achieved a partial response $(95 \% \mathrm{Cl}, 8.2-47.2 \%)$,stable disease was observed in 9 patients $(42.9 \%)$, and progressive disease was observed in 5 patients (23.8\%). The disease control rate was $66.7 \%$. The median time to progression and overall survivaltime were 3.7 months $(95 \% \mathrm{Cl}$, 2.3-6.5 months) and 7.3 months (95\% confidence interval, 4.9-12.1 months), respectively. Toxicity was generally mild, and the most common grade $3 / 4$ toxicities were neutro-penia $(14.3 \%)$, anemia $(14.3 \%)$, leucopenia $(9.5 \%)$, thrombocytopenia $(9.5 \%)$, anorexia $(9.5 \%)$, and diarrhea $(9.5 \%)$. There were no treatment-related deaths.

Conclusions: Monotherapy with S-1 is an effective and well-tolerated regimen for elderly patients with unresectable pancreatic cancer.

Key words: pancreatic cancer, chemotherapy, elderly patients, S-1

\section{INTRODUCTION}

Pancreatic cancer is one of the most common types of gastrointestinal cancer, and its prognosis remains extremely poor. Indeed, pancreatic cancer is the fifth leading cause of cancer death in Japan, the US and European countries (1). Of all the treatments available for pancreatic cancer, only resection offers the chance of a cure. However, owing to the high frequency of local extension and/or metastatic disease at the time of diagnosis, only a small minority of patients are candidates
Corresponding author:

Masahiko Inamori, M.D., Ph.D.

Department of Medical Education, Yokohama City University School of Medicine

3-9 Fukuura, Kanazawa-ku, Yokohama Kanagawa, 236-0004, Japan Telephone: +81-45-787-2626 Fax: +81-45-787-2620

E-mail: inamorim@yokohama-cu.ac.jp
Received: 30.03 .2018

Accepted: 15.05 .2018 
for curative resection. Moreover, surgery alone is limited, showing an unsatisfactory prognosis and a high incidence of postoperative recurrence. To improve the survival rate of patients with pancreatic cancer, effective non-surgical treatments are urgently needed.

Gemcitabine (GEM) has become the standard treatment for advanced pancreatic cancer because it improves overall survival (OS) compared with fluorouracil (2). Although various GEM-based combination regimens have been evaluated, only erlotinib combined with GEM showed a survival benefit over GEM alone, and that benefit wasmarginal (3).

The GEM-free combination regimen fluorouracil/ leucovorin plus irinotecan plus oxaliplatin (FOLFIRINOX) was recently demonstrated to have clear survival benefits relative to GEM for patients with metastatic pancreatic cancer with a performance status of 0 to 1 (4). However, because FOLFIRINOX has significant toxicity, this regimen requires close monitoring and must be limited to patients with a good performance status (5).

S-1 is a novel orally administered drug that is a combination of tegafur (FT), 5-chloro-2,4-dihydroxypyridine (CDHP), and oteracil potassium (Oxo) in a 1:0.4:1 molar concentration ratio $(6,7)$. Tegafur is hydroxylated and converted to 5-fluorouracil (5-FU) byhepatic microsomal enzymes. 5-Chloro-2,4-dihydroxypyridine is a competitive inhibitor of dihydropyrimidine dehydrogenase, which is involved in the degradation of 5-FU, and it acts to maintain efficacious concentrations of 5$\mathrm{FU}$ in the plasma and tumor tissues. Oteracil potassium, a competitive inhibitor of orotate phosphoribosyltransferase, inhibits the phosphorylation of 5-FU in the gastrointestinal tract, thereby reducing the serious gastrointestinal toxicity associated with 5-FU. S-1 shows broad activity against a variety of tumors and is currently approved in Japan for use in patients with non-smallcell lung cancer, gastric cancer, colorectal cancer, breast cancer, head and neck cancer, biliary tract cancer and pancreatic cancer (8-14).

Two phase II studies (15) of S-1 in untreated patients with metastatic pancreatic cancer demonstrated that this compound is an active and well-tolerated drug. Consequently, S-1 was approved for the treatment of pancreatic cancer in Japan in 2006. A randomized, open-label, three-arm, phase III study (GEST) (16) was subsequently reported, in which 834 chemotherapy naïve unresectable advanced pancreatic cancer patients with an Eastern Cooperative Oncology Group (ECOG) performance status (PS) of 0 to 1 were randomly assigned to receive GEM $\left(1,000 \mathrm{mg} / \mathrm{m}^{2}\right.$ on days 1,8 , and $15, \mathrm{q} 4 \mathrm{w}), \mathrm{S}-1$ (60/80/100 $\mathrm{mg} / \mathrm{d}$ based on body surface area (BSA) on days 1-28, q6w), or GS (GEM 1,000 mg/m² on days 1 and 8, plus S-160/80/100 mg/d based on BSA on days 1-14, q3w). S-1 was confirmed to be noninferior to GEM with respect to OS (9.7 months vs. 8.8 months, $\mathrm{P}<0.001)$. Grade $3 / 4$ hematologic toxicities were more common in the GEM arm than the S-1 arm (neutropenia, $41.0 \%$ vs. $8.8 \%$; thrombocytopenia, $11.0 \%$ vs. $1.5 \%$, respectively), whereas digestive toxicities were more common in the S-1 arm than in the GEM arm (anorexia, $11.4 \%$ vs. $7.3 \%$; diarrhea, $5.5 \%$ vs. $1.1 \%$, respectively). This trial was the first to demonstrate that oral S-1 has an efficacysimilar to that of GEMwith tolerable toxicity.

As the Japanese population grows older, more patients over the age of 70 are being diagnosed with unresectable pancreatic cancer. Indeed, increases in the incidence rate have been observed among both males and females 75 years or older. Thus, the application of chemotherapy for elderly patients with unresectable pancreatic cancer is an important issue. S-1appears advantageous because it has few side effects and can be administered on an outpatient basis. Additionally, S-1 offers the possibility of treating patients with conditions such as pancreatic cancer with poor prognosis without affecting their quality of life (QOL), making it particularly promising for the treatment of elderly patients with advanced pancreatic cancer $(17,18)$. However, very few reports have evaluated the effects of S-1on unresectable pancreatic cancer in a cohort of elderly patients (19).

Therefore, we conducted a phase II study of monotherapy with S-1 in elderly patients with advanced unresectable pancreatic cancer and assessed the efficacy and toxicity of this regimen.

\section{METHODS}

\section{End point}

The primary end point of this study was to determine the efficacy of monotherapy with S-1 in elderly patients with unresectable pancreatic cancer. The secondary end points were to assesstoxicity, time to progression (TTP), and survival.

\section{Patients}

The eligibility criteria were as follows: (1) pancreatic adenocarcinoma diagnosed by pathological examination or typical radiographic findings; (2) unresectable locally advanced or metastatic disease; (3) at least one measurable lesion; (4) 70-90 years of age; (5) no history of prior antitumor treatment, except resection; (6) a Karnofsky performance status (KPS) of 50-100 points; (7) an estimated life expectancy of at least 8 weeks; and 
(8) adequate renal function (normal serum creatinine and creatinine clearance $\geq 50 \mathrm{ml} / \mathrm{min}$ ), liver function (total bilirubin $<3.0 \mathrm{mg} / \mathrm{dl}$ and transaminase level $\leq 150$ $\mathrm{IU} / \mathrm{I}$ ) and bone marrow reserves (white blood cell (WBC) count $\geq 3,500 / \mathrm{mm}^{3}$, platelets $\geq 100,000 / \mathrm{mm}^{3}$, and hemoglobin $\geq 9.0 \mathrm{~g} / \mathrm{dl}$ ). The exclusion criteria were as follows: (1) under regular treatment with phenytoin, warfarin, or flucytosine; (2) severe complications such asa mental disorder, active infection, ileus, heart failure, liver failure, renal failure, active gastric or duodenal ulcer, massive pleural or abdominal effusion, or watery diarrhea; (3) active concomitant malignancy; or (4) pregnancy or lactation.

\section{Study treatment}

S-1 was administered orally at a dose of $40 \mathrm{mg} / \mathrm{m}^{2}$ twice daily after breakfast and dinner. Three initial doses were established based on BSA as follows: BSA $<1.25 \mathrm{~m}^{2}$, $80 \mathrm{mg} /$ day; $1.25 \mathrm{~m}^{2} \leq \mathrm{BSA}<1.50 \mathrm{~m}^{2}, 100 \mathrm{mg} /$ day; and $1.50 \mathrm{~m}^{2} \leq \mathrm{BSA}, 120 \mathrm{mg} /$ day. S-1 was administered for 28 days, followed by a 14-day rest period. This treatment course was repeated until the occurrence of disease progression, unacceptable toxicities were reached, or the patient refused to continue.

The occurrence of any grade 3 or higher severe hematologic toxicity or grade 2 or higher severe non-hematologic toxicity resulted in the temporary discontinuation of S-1 until the toxicity recovered to grade 1 or less; then, the S-1 dose was reduced by 20 $\mathrm{mg} /$ day in the next treatment cycle (minimum dose, 80 $\mathrm{mg} /$ day). If no toxicity occurred, shortening of the rest period to 7 days was allowed. If a rest period of more than 28 days was required due to toxicity, the patient was withdrawn from the study. Patients were not allowed to receive concomitant radiation therapy, chemotherapy, or hormonal therapy during the study.

\section{Response and toxicity evaluation}

The response after each course was evaluated according to the Response Evaluation Criteria in Solid Tumors (RECIST) guidelines (20). Physical examination, complete blood cell counts, biochemistry tests, and urinalyses were performed at least biweekly. Adverse events were evaluated according to the National Cancer Institute Common Toxicity Criteria, version 3.0 (21).

The response duration was calculated from the day of the first sign of a response until disease progression. TTP was calculated from the date of study entry until documented disease progression. The OS time was calculated from the date of study entry to the date of death or the last follow-up.

\section{Statistical analysis}

The number of patients required for the study was determined according to the optimal two-stage design. The threshold response rate and expected response rate were $10 \%$ and $30 \%$, respectively. The sample size of this trial was 21 patients ( $\alpha$ - and $\beta$-error probabilities of 0.05 and 0.2 , respectively). Time-related parameters were analyzed using the Kaplan-Meier methodin an intention-to-treat analysis.

\section{Ethical Approval}

The study was conducted in accordance with the principles of the Declaration of Helsinki. Written informed consent was obtained from all patients treated with S-1. This study was conducted with the approval of the hospital institutional review board. The study is registered with the UMIN Clinical Trials Registry under UMIN000006369.

\section{RESULTS}

\section{Patient characteristics}

A total of 21 patients with unresectable pancreatic cancer were enrolled in the study between August 2008 and June 2013. All patients were eligible and assessable for responses and adverse events. The patient characteristics are summarized in table 1. Fourteen males and 7 females with a median age of 76 years (range 70-84 years) were treated with S-1. Of the 21 patients, 18 had metastatic disease at the time ofenrollment in the study, whereas three patients were diagnosed with locally advanced disease. The liver was the most common site of metastasis (10 patients), followed by the distant lymph nodes (5 patients) and the lungs (two patients).

A total of 110 cycles of therapy were delivered, with a median of 2 cycles (range, 1-38). The initial administered dose of S-1 was $80 \mathrm{mg} /$ day in one patient, 100 $\mathrm{mg} /$ day in 12 patients and $120 \mathrm{mg} /$ day in 8 patients. Dose reduction was required in 7 patients for the following reasons: grade 4 anemia (one patient); grade 3 thrombocytopenia (two patients); grade 2 gastrointestinal toxicity, including anorexia, nausea and stomatitis (one patient each); and grade 2 rash (one patient). All patients were treated as outpatients.

\section{Efficacy}

Twenty-one patients were assessed for their response to treatment. These responses are shown in table 2. There was no case of complete remission (CR); however, 5 patients showed partial remission (PR). Nine 
Table 1 - Patient characteristics $(n=21)$

\begin{tabular}{|c|c|c|}
\hline Characteristics & Patients & $\%$ \\
\hline \multicolumn{3}{|l|}{ Gender } \\
\hline Male & 14 & 67 \\
\hline Female & 7 & 33 \\
\hline Median age, years (range) & \multicolumn{2}{|c|}{$76(70-84)$} \\
\hline \multicolumn{3}{|l|}{ Karnofsky performance status (\%) } \\
\hline 100 & 5 & 24 \\
\hline 90 & 9 & 43 \\
\hline 80 & 3 & 14 \\
\hline 70 & 4 & 19 \\
\hline \multicolumn{3}{|l|}{ First dose (mg) } \\
\hline 40 & 1 & 5 \\
\hline 50 & 12 & 57 \\
\hline 60 & 8 & 38 \\
\hline \multicolumn{3}{|l|}{ Stage } \\
\hline Locally advanced & 3 & 14 \\
\hline Metastatic & 18 & 86 \\
\hline History of pancreatectomy & 0 & 0 \\
\hline \multicolumn{3}{|l|}{ Sites of metastasis } \\
\hline Liver & 10 & 48 \\
\hline Distant lymph node & 5 & 24 \\
\hline Lung & 2 & 10 \\
\hline Peritoneum & 1 & 5 \\
\hline
\end{tabular}

Table 2 - Tumor responses

\begin{tabular}{lc}
\hline Tumor response & Total $\mathbf{( n = 2 1 )}$ \\
\hline Complete response (CR) & 0 \\
\hline Partial response (PR) & 5 \\
\hline Stable disease (SD) & 9 \\
\hline Progressive disease (PD) & 5 \\
\hdashline Not evaluated\# (NE) & 2 \\
\hline Response rate $(\%)$ & 23.8 \\
\hline Disease control rate $(\%)(C R+P R+S D)$ & 66.7 \\
\hline
\end{tabular}

\# Radiographic assessment was not determined

patients showed stable disease (SD), and 5 patients showed progressive disease (PD). The overall response rate was $23.8 \%(95 \% \mathrm{Cl}, 8.2-47.2 \%)$, and the disease control rate was $66.7 \%(95 \% \mathrm{Cl}, 43.0-85.4 \%)$. At the time of analysis, 19 of the 21 patients had died due to disease progression. The median TTP was 3.7 months, the OS was 7.3 months, and the one-year survival rate was $28.6 \%$ (fig. 1).

\section{Toxicity}

All 21 patients were evaluated for toxicity using the National Cancer Institute Common Toxicity Criteria version 3.0. Hematologic and non-hematologic toxicities

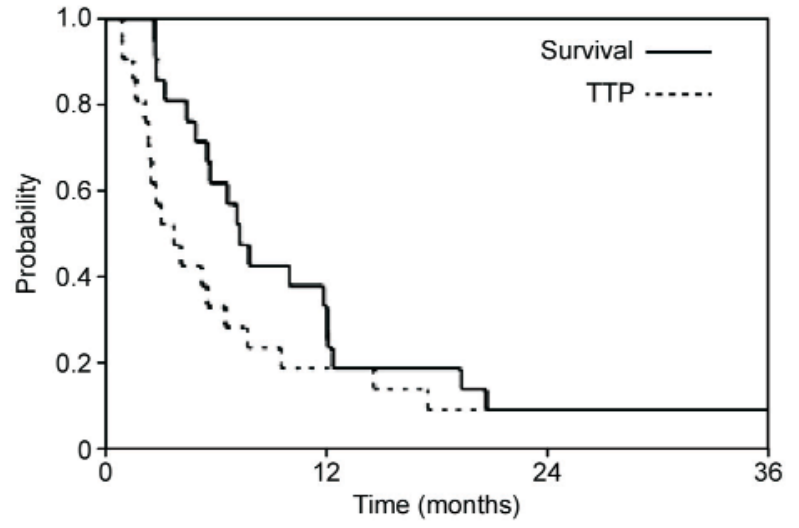

Figure 1 - Kaplan-Meier curves for overall survival (OS; solid line) and time to progression (TTP; dotted line)

are summarized in table 3. Treatment was generally well tolerated throughout the study. Although hematologic and gastrointestinal toxicities were common, most of these toxicities were mild and transient. Grade 3/4 anemia and neutropenia each occurred in three patients (14.3\%), and grade 3 leucopenia, thrombo-cytopenia, anorexia, and diarrhea each occurred in two patients (9.5\%). Although one patient died within 8 weeks of study enrollment due to rapid disease progression, no treatment-related deaths were observed. 
Table 3 - Treatment-related adverse events $(n=21)$ : Worst grade reported during treatment period. AST = aspartate aminotransferase; ALT $=$ alanine aminotransferase

\begin{tabular}{|c|c|c|c|c|c|c|}
\hline \multirow[t]{2}{*}{ Toxicity } & \multicolumn{4}{|c|}{ Grade } & \multirow{2}{*}{$\begin{array}{c}\text { Grade1-4 } \\
\%\end{array}$} & \multirow{2}{*}{$\begin{array}{c}\text { Grade 3-4 } \\
\%\end{array}$} \\
\hline & 1 & 2 & 3 & 4 & & \\
\hline \multicolumn{7}{|l|}{ Hematological toxicity } \\
\hline Leukopenia & 5 & 3 & 2 & 0 & 47.6 & 9.5 \\
\hline Neutropenia & 2 & 2 & 3 & 0 & 33.3 & 14.3 \\
\hline Hemoglobin reduction & 3 & 8 & 2 & 1 & 66.7 & 14.3 \\
\hline Thrombocytopenia & 7 & 1 & 2 & 0 & 47.6 & 9.5 \\
\hline \multicolumn{7}{|l|}{ Non-hematological toxicity } \\
\hline Anorexia & 5 & 5 & 2 & 0 & 57.1 & 9.5 \\
\hline Nausea & 4 & 2 & 0 & 0 & 28.6 & 0 \\
\hline Vomiting & 1 & 0 & 1 & 0 & 9.5 & 4.8 \\
\hline Diarrhea & 2 & 0 & 2 & 0 & 19.0 & 9.5 \\
\hline Stomatitis & 1 & 3 & 0 & 0 & 19.0 & 0 \\
\hline Rash & 2 & 3 & 0 & 0 & 23.8 & 0 \\
\hline Pigmentation & 4 & 0 & 0 & 0 & 19.0 & 0 \\
\hline Colitis & 0 & 0 & 1 & 0 & 4.8 & 4.8 \\
\hline Biliary tract infection & 0 & 4 & 0 & 0 & 19.0 & 0 \\
\hline Fatigue & 1 & 0 & 0 & 0 & 4.8 & 0 \\
\hline Thrombophlebitis & 1 & 0 & 0 & 0 & 4.8 & 0 \\
\hline AST elevation & 3 & 1 & 0 & 0 & 19.0 & 0 \\
\hline ALT elevation & 1 & 1 & 0 & 0 & 9.5 & 0 \\
\hline Total bilirubin elevation & 2 & 1 & 1 & 0 & 19.0 & 4.8 \\
\hline Creatinine elevation & 4 & 0 & 0 & 0 & 19.0 & 0 \\
\hline
\end{tabular}

\section{DISCUSSION}

Based on the latest cancer statistics put forth by the National Cancer Research Center, pancreatic cancer is the fifth leading cause of cancer death in Japan (22). Additionally, the prevalence of pancreatic cancer has increased in both men and women aged 75 years or older. Therefore, studies performed in elderly populations are necessary, particularly as the incidence of pancreatic cancer in this group is expected to increase in the future.

For pancreatic cancer patients with a poor prognosis, the effect of treatments on QOL is very important. We decided to test S-1 therapy in elderly pancreatic cancer patients for several reasons. First, S-1 therapy is simple and presents few side effects; thus, we could treat these patients as outpatients. Second, the GEST trial (16) revealed that GEM therapy and S-1 therapy were similarly effective, which allowed us to choose S-1 therapy as a first-line treatment. In our trial, all patients could receive S-1 therapy in the outpatient department and were able to maintain their QOL.

A retrospective study of GEM therapy involving 39 elderly pancreatic cancer patients (23) showed that $59 \%$ of the patients could be treated with full-dose GEM therapy. The grade $3 / 4$ side effects included neutropenia (38\%), thrombopenia (28\%) and anemia
(18\%). The TTP was 7 months, and the OS was 10 months. In summary, the selected elderly patients could obtain benefits from GEM therapy similar to those achieved in younger patients.

Two prospective studies of S-1 therapy for younger pancreatic cancer patients have been reported. First, Ueno et al. (24) showed in an early phase II trial that the response rate was $21.1 \%$, disease control rate was $73.7 \%$, OS was 5.6 months, and 1-year survival rate was $15.8 \%$. The grade $3 / 4$ side effects included anorexia $(15.8 \%)$, nausea $(15.8 \%)$, ileus $(15.8 \%)$, neutropenia (5.3\%) and anemia (5\%). Second, Okusaka et al. (25) showed in a late phase II trial that the response rate was $37.5 \%$, disease control rate was $67.5 \%$, OS was 9.2 months, 1-year survival rate was $32.5 \%$, and TTP was 3.7 months. The grade $3 / 4$ side effects included anorexia (12.5\%), nausea (7.5\%), neutropenia (12.5\%) and anemia (5.0\%).

In contrast, our study showed that the response rate was $23.8 \%$, disease control rate was $66.7 \%$, OS was 7.3 months, 1 -year survival rate was $28.6 \%$, and TTP was 3.7 months. The grade $3 / 4$ side effects included anorexia $(9.5 \%)$, nausea (0\%), diarrhea (9.5\%), leukopenia (9.5\%), neutropenia (14.3\%), thrombopenia (9.5\%) and anemia (14.3\%).

Variables contributing to our favorable results were the selective registration criteria, a good KPS ( $\geq 70$ in all 
patients and $\geq 80$ in $17 / 21$ patients) and the fact that most of the patients were treated with second-line therapy (12/19 patients).

Myelosuppression was observed as a side effect in many patients, which was not observed in previous studies. We speculate that the bone marrow of the elderly patients could not with stand long-term chemotherapy. After S-1 therapy was repeated for more than 10 courses, one patient presented with neutropenia, two with anemia and one with thrombopenia. One patient treated with 42 courses of S-1 therapy presented with the side effects of grade 4 anemia and grade 3 neutropenia; however, this patient's tumor showed only aPR.

In a previous S- 1 trial in Japan, there were few reports of diarrhea. In contrast, diarrhea is commonly reported in Western patients on S-1 therapy. In this study, we found that grade $3 / 4$ diarrhea occurred in $9.5 \%$ of patients. One patient with digestive organ toxicity (anorexia, diarrhea and vomiting with a grade of 3 or 4) was withdrawn from treatment after 1 or 2 courses of S-1 therapy.

\section{CONCLUSION}

In conclusion, monotherapy with S-1 may be an effective and well-tolerated regimen for elderly patients with unresectable pancreatic cancer. Because this study had a small sample size and may have been subject tobias, it was not possible to make simple comparisons between our results and those of previous studies. Thus, a large-scale prospective study is needed to conclusively demonstrate the safety and efficacy of S-1 in elderly patients, which we hope to conductin the future.

\section{Conflicts of Interest and Source of Funding}

All author has no conflict of interest. No financial support was obtained for this study.

\section{Acknowledgements}

Special thanks to the medical staff of Chigasaki Municipal Hospital.

\section{REFERENCES}

1. Matsuno S, Egawa S, Fukuyama S, Motoi F, Sunamura M, Isaji S, et al. Pancreatic Cancer Registry in Japan: 20 years of experience. Pancreas. 2004;28(3):219-30.

2. Burris HA 3rd, Moore MJ, Andersen J, Green MR, Rothenberg ML, Modiano MR, et al. Improvements in survival and clinical benefit with gemcitabine as first-line therapy for patients with advanced pancreas cancer: a randomized trial. J Clin Oncol. 1997;15(6):2403-13.
3. Moore MJ, Goldstein D, Hamm J, Figer A, Hecht JR, Gallinger S, et al. Erlotinib plus gemcitabine compared with gemcitabine alone in patients with advanced pancreatic cancer: a phase III trial of the National Cancer Institute of Canada Clinical Trials Group. J Clin Oncol. 2007;25(15):1960-6. Epub 2007 Apr 23.

4. Conroy T, Desseigne F, Ychou M, Bouché 0 , Guimbaud $\mathrm{R}$, Bécouarn $\mathrm{Y}$, et al. FOLFIRINOX versus gemcitabine for metastatic pancreatic cancer. N Engl J Med. 2011; 364(19):181725. doi: 10.1056/ NEJMoa1011923.

5. Saif MW, Chabot J. Chemotherapy: metastatic pancreatic cancer - is FOLFIRINOX the new standard? Nat Rev Clin Oncol. 2011;8(8):452-3. doi: 10.1038/nrclinonc.2011.107.

6. Tatsumi K, Fukushima M, Shirasaka T, Fujii S. Inhibitory effects of pyrimidine, barbituric acid and pyridine derivatives on 5-fluorouracil degradation in rat liver extracts. Jpn J Cancer Res. 1987;78(7):748-55

7. Shirasaka T, Shimamoto $Y$, Fukushima M. Inhibition by oxonic acid of gastrointestinal toxicity of 5-fluorouracil without loss of its antitumor activity in rats. Cancer Res. 1993;53(17):4004-9.

8. Hubbard JM, Grothey A, Sargent DJ. Systemic therapy for elderly patients with gastrointestinal cancer. Clin Med Insights Oncol. 2011:5:89-99. doi: 10.4137/CM0.S6983. Epub 2011 Apr 25.

9. Ueno H, Okusaka T, Ikeda M, Takezako Y, Morizane C. Phase II study of S-1 in patients with advanced biliary tract cancer. $\mathrm{Br} \mathrm{J}$ Cancer. 2004;91(10):1769-74.

10. Grande C, Quintero G, Candamio S, París Bouzas L, Villanueva MJ, Campos B, et al. Biweekly XELOX (capecitabine and oxaliplatin) as first-line treatment in elderly patients with metastatic colorectal cancer. Journal of Clinical OncologyJ Geriatr Oncol. 2013;4(2):114-21. doi: 10.1016/j.jgo.2013.01.001. Epub 2013 Feb 16.

11. Hutchins LF, Unger JM, Crowley JJ, Coltman CA Jr, Albain KS. Underrepresentation of patients 65 years of age or older in cancer-treatment trials. N Engl J Med. 1999 Dec 30;341(27): 2061-7.

12. Kahn KL, Adams JL, Weeks JC, Chrischilles EA, Schrag D, Ayanian JZ et al. Adjuvant chemotherapy use and adverse events among older patients with stage III colon cancer. JAMA. 2010;303(11): 1037-45. doi: 10.1001/jama.2010.272.

13. Neugut Al, Matasar M, Wang X, McBride R, Jacobson JS, Tsai $W Y$, et al. Duration of adjuvant chemo-therapy for colon cancer and survival among the elderly. J Clin Oncol. 2006;24(15):236875. Epub 2006 Apr 17.

14. Köhne CH, Folprecht G, Goldberg RM, Mitry E, Rougier P. Chemotherapy in elderly patients with colorectal cancer. Oncologist. 2008;13(4):390-402. doi: 10.1634/theoncologist. 2007-0043.

15. Nakamura K, Yamaguchi T, Ishihara T, Sudo K, Kato H, Saisho H. Phase II trial of oral S-1 combined with gemcitabine in metastatic pancreatic cancer. Br J Cancer. 2006;94(11):1575-9.

16. Ueno H1, Ioka T, Ikeda M, Ohkawa S, Yanagimoto H, Boku N, et al. Randomized phase III study of gemcitabine plus S-1, S-1 alone, or gemcitabine alone in patients with locally advanced and metastatic pancreatic cancer in Japan and Taiwan: GEST study. J Clin Oncol. 2013;31(13):1640-8. doi: 10.1200/JC0.2012.43. 3680. Epub 2013 Apr 1.

17. Morizane C, Okusaka T, Ito $\mathrm{Y}$, Ueno H, Ikeda M, Takezako $\mathrm{Y}$, et al. Chemoradiotherapy for locally advanced pancreatic carcinoma in elderly patients. Oncology. 2005;68(4-6):432-7. Epub 2005 Jul 14.

18. Miyamoto DT, Mamon HJ, Ryan DP, Willett CG, Ancukiewicz M, Kobayashi WK, et al. Outcomes and tolerability of chemoradiation therapy for pancreatic cancer patients aged 75 years or older. Int J Radiat Oncol Biol Phys. 2010;77(4):1171-7. doi: 10.1016/j.jijrobp. 2009.06.020. Epub 2009 Sep 30.

19. Aoyama T, Katayama Y, Murakawa M, Atsumi Y, Yamaoku K, Kanazawa A, et al. Safety and feasibility of S-1 adjuvant chemotherapy for pancreatic cancer in elderly patients. Cancer Chemother Pharmacol. 2015;75(6):1115-20. doi: 10.1007/ s00280-015-2736-5. Epub 2015 Mar 29.

20. World Health Organization. WHO handbook for reporting results of cancer treatment. World Health Organization: Geneva; 1979 
21. National Cancer Institute. Common toxicity criteria (version 3.0) Division of Cancer Treatment and Diagnosis; 2003

22. Anazawa T, Miyata H, Gotoh M. Cancer registries in Japan: National Clinical Database and site-specific cancer registries. Int J Clin Oncol. 2015;20(1):5-10. doi: 10.1007/s10147-014-07574. Epub 2014 Nov 8.

23. Locher C, Fabre-Guillevin E, Brunetti F, Auroux J, Delchier JC Piedbois $P$, et al. Fixed-dose rate gemcitabine in elderly patients with advanced pancreatic cancer: an observational study. Crit
Rev Oncol Hematol. 2008;68(2):178-82. doi: 10.1016/j. critrevonc.2008. 06.010. Epub $2008 \mathrm{Jul} 30$.

24. Ueno H, Okusaka T, Ikeda M, Takezako Y, Morizane C. An early phase II study of S-1 in patients with metastatic pancreatic cancer. Oncology. 2005;68(2-3):171-8. Epub 2005 Jul 4.

25. Okusaka T, Funakoshi A, Furuse J, Boku N, Yamao K, Ohkawa S, Saito $\mathrm{H}$. A late phase II study of S-1 for metastatic pancreatic cancer. Cancer Chemother Pharmacol. 2008;61(4):615-21. Epub 2007 May 23. 